\title{
Sucrose-induced translational repression of plant bzIP-type transcription factors
}

\author{
A. Wiese, N. Elzinga, B. Wobbes ${ }^{1}$ and S. Smeekens ${ }^{2}$ \\ Molecular Plant Physiology, Utrecht University, Padualaan 8, 3584 CH Utrecht, The Netherlands
}

\begin{abstract}
Sugars as signalling molecules exert control on the transcription of many plant genes. Sugar signals also alter mRNA and protein stability. Increased sucrose concentrations specifically repress translation of the S-class basic region leucine zipper (bZIP) type transcription factor AtbZIP11/ATB2. This sucrose-induced repression of translation (SIRT) depends on translation of a highly conserved upstream open reading frame (UORF) in the $5^{\prime}$ UTR of the gene. This conserved uORF is exclusively encoded in $5^{\prime}$ UTRs of several plant S-class bZIP transcription factors. Arabidopsis homologues of ATB2/AtbZIP11, which harbour the conserved uORF, also show SIRT. Therefore, SIRT emerges as a general sucrose translational control mechanism of a group of transcription factors. SIRT might be part of a sucrose-specific signalling pathway, controlling expression of plant bzıP transcription factor genes.
\end{abstract}

\section{Sucrose sensing in plants}

Plants are autotrophic and capable of synthesizing sugars driven by light energy. This process is feedback-inhibited by its end product sugars that repress the transcription of photosynthesis related genes [1]. Sugars are important energy carriers and building blocks for all organisms. Metabolism, growth and development of plants are adjusted to the sugar availability by changing expression of genes. Such changes are mediated by transcriptional and post-transcriptional mechanisms. Sugars change gene expression via control of mRNA stability, mRNA translation or protein stability [2-4].

Plants acquired elaborate systems to sense the availability of sugars, distinguishing between hexoses and the storage and transport sugar, sucrose. Generally hexoses are thought to control metabolism and growth, whereas sucrose regulates differentiation and storage [5]. Sucrose was shown to specifically control gene expression. Sucrose control operates separately from the sucrose breakdown products glucose and fructose. In the case of the sucrose induction of a fructan biosynthesis gene (6-SFT) sucrose control is stimulated by the addition of invertase inhibitor 2,5-dideoxy-2,5-imino-Dmannitol [6]. Specifically sucrose controlled genes encode, for example, patatin, UDP-glucose pyrophosphorylase, protonsucrose symporters (LeSUT2, BvSUT1) and a sucrose: fructan-6-fructosyl-transferase (6-SFT) in barley [6-9]. Nonmetabolizable analogues of sucrose, like palatinose and turanose, were used to test if disaccharide-sensing is independent from sucrose metabolism. Such analogues mimic the

Key words: bzlP, plant gene expression, sucrose, sugar sensing, transcription factor, translational control, upstream open reading frame (UORF).

Abbreviations used: AAP, arginine attenuator peptide; bzIP, basic region leucine zipper; GCN4 general control non-derepressible 4; IRES, internal ribosome entry sites; SAM, S-adenosyl-L methionine; SIRT, sucrose-induced repression of translation; UORF, upstream open reading frame; SC-uORF, sucrose-controlled UORF; UTR, untranslated region.

'Present address: Nickerson-Zwaan BV, P.O. Box 28, 4920 AA Made, The Netherlands.

${ }^{2}$ To whom correspondence should be addressed (email j.c.m.smeekens@bio.uu.nl). sucrose effects on the expression of the vine hexose transporter VvHT1, the $\alpha$-amylase in barley embryos and on the activation of the ADP-glucose pyrophosphorylase in potato [10-12], but also activated other signalling pathways [13]. Detailed information on signalling pathways mediating sucrose or hexose signals in plants is currently lacking [14]. Hexokinase has been identified as a sensor for hexoses [15], but other hexokinase-independent pathways exist, which possibly include hexose transporters as sensor molecules [14]. Sucrose-specific signalling pathways are currently not understood. A sensor function for sucrose transporters with extended cytoplasmic loops was proposed but has not been experimentally confirmed $[16,17]$. Sucrose-mediated repression of the sugar beet sucrose transporter gene BvSUT1 and induction of 6-STF in barley were shown to involve protein phosphorylation and dephosphorylation by the use of protein kinase and phosphatase inhibitors [18]. The sucroseinduced redox activation of the AGPase in potato depends on SNF-like kinase 1 [10]. Sucrose-insensitive mutants with impaired sucrose control would be helpful to gain insight into the specific sucrose signalling pathways, but such mutants have not been described so far.

\section{Metabolite-controlled translation}

Translational control is an important general mechanism for regulating gene expression. Up to $20 \%$ of the expressed genes can be subject to translational control [19]. Translational control overrides transcriptional control and allows fine-tuning of gene expression. Inhibition or induction of translation is a rapid flexible way to respond to stimuli, because it is independent of transcription and mRNA processing.

Deprivation of nutrients and other stress conditions generally inhibits translation and under such conditions only specific mRNAs are translated [20]. Metabolite-mediated translational control has been particularly well studied for the 
yeast bZIP transcription factor GCN4 (general control nonderepressible 4). Translation of this gene is induced by amino acid starvation. Such lack of amino acids globally inhibits protein synthesis [21]. Translation of an arginine/lysine transporter (Cat-1) in mammalians is induced by amino acid starvation as well [22].

Elevated concentrations of nutrients and metabolites also control translation. For example, arginine-excess represses the translation of genes encoding the small subunit of arginine-specific carbamoyl phosphate synthetase (CPA-1 and arg-2) in fungi [23]. This is a product feedback inhibition on the synthesis of the enzyme, since carbamoyl phosphate is employed in both pyrimidine and arginine biosynthesis [24]. Polyamines inhibit translation of the mammalian and plant S-adenosyl-L-methionine (SAM) decarboxylase. In transgenic tobacco, removal of the control element in the mRNA relieved translational control and resulted in severe growth perturbations because of unrepressed translation of the enzyme and increased accumulation of polyamines [25]. This clearly demonstrated the physiological importance of metabolite-induced translational regulation in plants.

\section{Mechanisms of translational control}

The elements mediating translational control are mostly located in the untranslated regions (UTRs) of the mRNA and act in cis. A more general control of translational efficiency is mediated by the $5^{\prime}$ cap structures as well as the $3^{\prime}$ poly(A) tails. Various features were shown to be important for translational control. These include the length of the 5' UTR, secondary structures of the mRNA, upstream start codons (uAUGs) or open reading frames ( $\mathrm{uORF}$ ) and internal ribosome entry sites (IRES) [26].

Translational control through trans-acting elements was shown for micro-RNAs and regulatory proteins recognizing binding sites in the mRNA $[26,27]$.

A direct interaction of a metabolite with the secondary structure of the mRNA, that results in translational inhibition, has been shown in prokaryotes and was also suggested to exist in Arabidopsis [28].

The above-mentioned examples for metabolite controlled translation mostly involve uORFs. The majority of translation events start with binding of the $40 \mathrm{~S}$ ribosomal subunit to the mRNA cap-structure and subsequent scanning of the sequence for the 5'-proximal AUG codon. The initiation codon selection depends on the context of the start codon. Initiation at an uORF usually affects the translation efficiency of the downstream open reading frame. Inhibition or stimulation of downstream translation may be regulated dependent or independent of the amino acid sequence of the UORF.

Translational control of the yeast bZIP transcription factor GCN4 was shown to be independent of the amino acid sequences of the encoded short uORFs in the $5^{\prime}$ UTR. This mechanism involves the reinitiation of the ribosomes after translation of the GCN4 uORF1 [29].

Amino acid starvation induces translation of the mammalian cat1 gene. Translation of the uORF stimulates the initiation at the main ORF by opening an inhibitory structure in the $5^{\prime}$ UTR and induction of a conformational change that yields an active IRES and induction of translation. This happens independent of the amino acid sequence of the $\mathrm{uORF}$ [22].

Ribosome stalling mechanisms were observed to depend on the encoded amino acid sequence of the UORF, which is thought to interact with RNA or protein components of the translational machinery during or at the end of their synthesis [30]. The arginine-regulated carbamoyl-phosphate synthase genes in fungi, encode a conserved upstream open reading frame in their $5^{\prime}$ UTRs called the arginine attenuator peptide (AAP). Here a direct interaction between the nascent $\mathrm{AAP}$ and arginine is thought to mediate ribosome stalling at the $3^{\prime}$-end of the uORF and subsequent repression of further ribosome scanning and translation of the carbamoyl-phosphate synthase downstream [31]. A similar mechanism is proposed for the mammalian SAM decarboxylase genes [32]. Several amino acids of the short, conserved uORF encoding the hexapeptide MAGDIS are essential for the polyamineinduced repression of translation. Plant SAM decarboxylase genes also encode a highly conserved uORF of 50 to 54 amino acids ('small uORF') overlapped by a short uORF of two to four amino acids ('tiny uORF'), not showing any similarity to the mammalian uORF [33]. Removal of the small uORF caused loss of the polyamine repression of translation.

More sequence-dependent and conserved uORFs have been identified, but so far conserved domains possibly involved in interaction with the translational apparatus or RNA have not been identified [34].

\section{Translational control of S-class bzIP transcription factors}

Transcription of the S-class bZIP transcription factor ATB2/ AtbZIP11 [35] is stimulated by sugars and light, but the subsequent translation of ATB2/AtbZIP11 can be repressed by elevated sucrose concentrations [3].

ATB2/AtbZIP11 mRNA is transcribed with a very long $5^{\prime}$ UTR, which is indispensable for the sucrose repression [3]. In total, AtbZIP11 encodes four uORFs in the $5^{\prime}$ UTR. Starting from the $5^{\prime}$-end of the mRNA, these overlapping $\mathrm{UORF}$ s are designated uORF1 (18 amino acids), uORF2 (42 amino acids), uORF3 (five amino acids) and uORF4 (19 amino acids). Disruption of the coding sequence of uORF2 by a stop codon in planta annihilates the sucroseinduced repression of translation (SIRT). Therefore, SIRT depends on translation of uORF2. This so-called sucrose control uORF (SC-uORF) can be translated in vitro in the native $5^{\prime}$ UTR context, showing that its start codon is indeed a signal for initiation [36].

Four other Arabidopsis S-class bZIP transcription factors AtbZIP2, AtbZIP44, AtbZIP53 and AtbZIP1 harbour a long $5^{\prime}$ UTR with a conserved SC-uORF (Figure 1). The five 'ATB2-type' genes form a subgroup of the Arabidopsis S-class of bZIP transcription factors [35] that consists of 17 members.

SIRT was also shown for AtbZIP2 and depends on the presence of its $5^{\prime}$ UTR harbouring the SC-uORF (Figure 2). 
Figure 1 Alignment of the Arabidopsis SC-uORFs

Black box, white letter, 100\% identity; dark grey box, white letter, 80\% identity; grey box, black letter, 60\% identity.

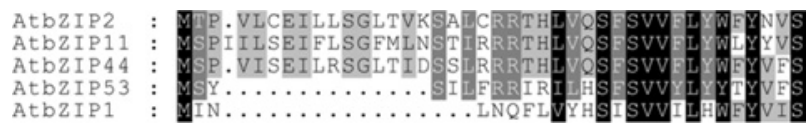

Figure 2 Translational control of AtbzIP2

Expression of AtbzIPz:GUS. Comparison of GUS activity (black filled circles) and relative expression of the GUS mRNA (grey bars), determined in quantitative real-time PCR. (A) Expression of the GUS marker in the AtbzIP2 context, (B) GUS expression resulting from deletion of the 5' UTR. Seedlings were grown for 5 days in liquid culture, half strength MS with sucrose, added in indicated concentrations.
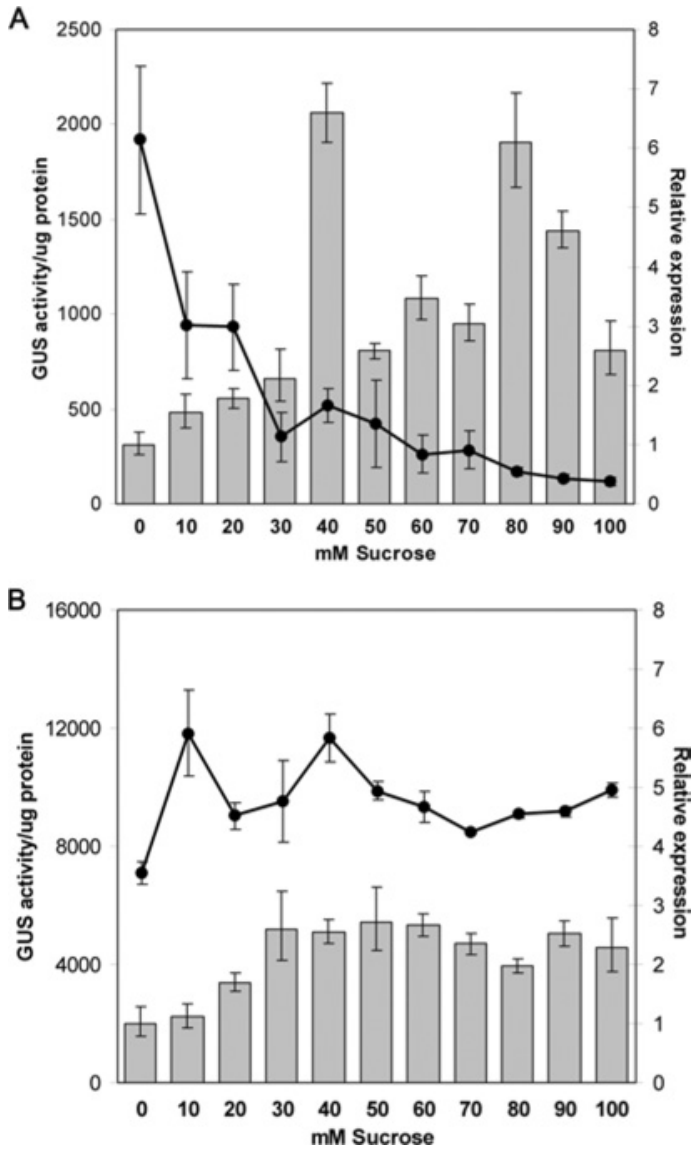

In 5-day-old seedlings, activity of $\beta$-glucuronidase (GUS) embedded in the AtbZIP2 gene is repressed with increasing sucrose concentration, whereas the GUS mRNA in these seedlings does not respond to the sucrose concentration (Figure 2A). Transgenic seedlings expressing the same construct with deletion of the AtbZIP2 5' UTR do not repress the GUS activity (Figure 2B). Therefore, it is expected that all members of the ATB2-type subgroup are regulated by SIRT in the same manner [36].

Other dicotyledonous and monocotyledonous plants species reveal SC-uORFs exclusively upstream of S-class
bZIP transcription factors. The conserved SC-uORFs encode 41-53 amino acids long peptides or shorter, 22-32 amino acid long peptides. Both sizes are found in and between species. In Arabidopsis, three long and two short SC-uORFs are encoded (Figure 1). Truncation of the AtbZIP11 Sc-uORF, artificially creating short $\mathrm{uORF}$ comparable to the natural ones, demonstrated their repressive activity [36].

Some of the SC-uORF encoding bZIP transcription factor genes have been characterized in terms of transcriptional activity and tissue specificity. The promoter of AtbZIP11 mediates expression in the vascular tissues of seedlings and young vegetative tissues and in funiculi of fertilized ovules [3]. By ubiquitous expression of the GUS marker gene, controlled by the ATB2/AtbZIP11 5' UTR it was shown that the sucrose repression system functions in all tissues of the seedling beyond the tissue-specific vascular expression pattern of $A T B 2 / A t b Z I P 11$. Thus SIRT can act on all bZIP transcription factor genes harbouring the SC-uORF with various expression patterns [36].

\section{Physiological consequences of SIRT}

The sucrose concentration in plants changes 5-10-fold during the diurnal cycle [37]. Concentrations of sucrose also change substantially during development. Gradients of hexose and sucrose have been shown during cotyledon differentiation of faba bean embryos or during rape seed development $[38,39]$. SIRT could be a mechanism allowing flexible responses of gene expression to sucrose changes locally and during time.

The high sensitivity of the system as shown for AtbZIP2 with almost 50\% repression in seedlings grown in low $(10 \mathrm{mM})$ sucrose concentrations (Figure 2), indicates the relevance of SIRT and its high responsiveness to low physiological sucrose concentrations.

The specific expression pattern of ATB2/AtbZIP11 with vascular association as well as the translational regulation by sucrose suggests its involvement in the resource allocation to newly established sinks [3]. The GNC4 transcription factor in yeast is translationally repressed by amino acids and controls the expression of amino acid biosynthesis genes. In analogy with this system, possibly target genes of the ATB2type transcription factors could be involved in the sucrose synthesis, breakdown or transport.

Photosynthesis results in increased sucrose levels [40] and the expression of ATB2/AtbZIP11 gene is light induced [41]. In the light, AtbZIP11 would thus be active until increased sucrose accumulation inhibits its translation. Other S-class transcription factors harbouring the SC-uORF are induced by various stimuli like low temperature, salt stress, hormones, pathogen attack, hypo-osmolarity or senescence [42-46]. These conditions result in changes in sucrose concentration that in turn affects translation of bZIP proteins and by this control expression of target genes. Thus transcription of ATB2-type bZIP transcription factors is regulated by different stimuli, but translation is then equally overridden by SIRT, responding to prevailing sucrose concentrations. So far the only proposed target gene of the ATB2-type group is the 
Arabidopsis proline dehydrogenase gene, which is involved in the breakdown of the osmolyte proline [43].

bZIP transcription factor act as homo- or heterodimers. Specific interaction of S-class bZIP transcription factors has been shown with C-class bZIP transcription factors [47]. The sucrose repression of the S-class bZIP transcription factors will result in changes in their abundance and by this the composition of such heterodimers as well. A variety of mechanisms are involved in controlling the activity of bZIP transcription factors, including transcription, translation, post-translational modifications and homo- or heterodimerization with other transcription factors. Such a system allows a flexible, multi-responsive regulation of bZIP target genes of which SIRT represents an important part.

In conclusion we propose SIRT to act on a subset of plant S-class bZIP transcription factors encoding the SC-uORF. Interaction of several control mechanisms allows these regulatory bZIP genes to respond in a flexible way to rapidly changing stimuli that affect sucrose levels in cells or tissues.

SIRT might be an essential part of a sucrose-specific signalling pathway. Target genes of the SC-uORF controlled bZIP transcription factors and their interacting partners will be sucrose responsive.

\section{References}

1 Sheen, J., Zhou, L. and Jang, J.C. (1999) Curr. Opin. Plant Biol. 2, 410-418 2 Chan, M.T. and Yu, S.M. (1998) Plant J. 15, 685-695

3 Rook, F., Gerrits, N., Kortstee, A., van Kampen, M., Borrias, M., Weisbeek, P. and Smeekens, S. (1998) Plant J. 15, 253-263

4 Yanagisawa, S., Yoo, S.D. and Sheen, J. (2003) Nature (London) 425 521-525

5 Wobus, U. and Weber, H. (1999) Biol. Chem. 380, 937-944

6 Muller, J., Aeschbacher, R.A., Sprenger, N., Boller, T. and Wiemken, A. (2000) Plant Physiol. 123, 265-274

7 Jefferson, R., Goldsbrough, A. and Bevan, M. (1990) Plant Mol. Biol. 14 995-1006

8 Wenzler, H., Mignery, G., Fisher, L. and Park, W. (1989) Plant Mol. Biol. 13, 347-354

9 Ciereszko, I., Johansson, H. and Kleczkowski, L.A. (2001) Biochem. J. 354, 67-72

10 Tiessen, A., Prescha, K., Branscheid, A., Palacios, N., McKibbin, R., Halford, N.G. and Geigenberger, P. (2003) Plant J. 35, 490-500

11 Loreti, E., Alpi, A. and Perata, P. (2000) Plant Physiol. 123, 939-948

12 Atanassova, R., Leterrier, M., Gaillard, C., Agasse, A., Sagot, E., Coutos-Thevenot, P. and Delrot, S. (2003) Plant Physiol. 131, 326-334

13 Roitsch, T., Balibrea, M.E., Hofmann, M., Proels, R. and Sinha, A.K. (2003) J. Exp. Bot. 54, 513-524

14 Smeekens, S. (2000) Annu. Rev. Plant Physiol. Plant Mol. Biol. 51, 49-81

15 Moore, B., Zhou, L., Rolland, F., Hall, Q., Cheng, W.H., Liu, Y.X., Hwang, I., Jones, T. and Sheen, J. (2003) Science 300, 332-336
16 Lalonde, S., Boles, E., Hellmann, H., Barker, L., Patrick, J.W., Frommer, W.B. and Ward, J.M. (1999) Plant Cell 11, 707-726

17 Barth, I., Meyer, S. and Sauer, N. (2003) Plant Cell 15, 1375-1385

18 Ransom-Hodgkins, W.D., Vaughn, M.W. and Bush, D.R. (2003) Planta 217, 483-489

19 Garcia-Sanz, J.A., Mikulits, W., Livingstone, A., Lefkovits, I. and Mullner, E.W. (1998) FASEB J. 12, 299-306

20 Bailey-Serres, J. (1999) Trends Plant Sci. 4, 142-148

21 Hinnebusch, A.G. (1997) J. Biol. Chem. 272, 21661-21664

22 Yaman, I., Fernandez, J., Liu, H., Сaprara, M., Komar, A.A., Koromilas, A.E., Zhou, L., Snider, M.D., Scheuner, D., Kaufman, R.J. and Hatzoglou, M. (2003) Cell 113, 519-531

23 Wang, Z., Gaba, A. and Sachs, M.S. (1999) J. Biol. Chem. 274 37565-37574

24 Holden, H.M., Thoden, J.B. and Raushel, F.M. (1999) Cell Mol. Life Sci. 56 507-522

25 Hanfrey, C., Franceschetti, M., Mayer, M.J., Illingworth, C. and Michael, A.J. (2002) J. Biol. Chem. 277, 44131-44139

26 Wilkie, G.S., Dickson, K.S. and Gray, N.K. (2003) Trends Biochem. Sci. 28, 182-188

27 Aukerman, M.J. and Sakai, H. (2003) Plant Cell 15, 2730-2741

28 Sudarsan, N., Barrick, J.E. and Breaker, R.R. (2003) RNA 9, 644-647

29 Gaba, A., Wang, Z., Krishnamoorthy, T., Hinnebusch, A.G. and Sachs, M.S. (2001) EMBO J. 20, 6453-6463

30 Vilela, C. and McCarthy, J.E. (2003) Mol. Microbiol. 49, 859-867

31 Wang, Z., Gaba, A. and Sachs, M.S. (1999) J. Biol. Chem. 274, 37565-37574

32 Mize, G.J., Ruan, H., Low, J.J. and Morris, D.R. (1998) J. Biol. Chem. 273, 32500-32505

33 Franceschetti, M., Hanfrey, C., Scaramagli, S., Torrigiani, P., Bagni, N., Burtin, D. and Michael, A.J. (2001) Biochem. J. 353, 403-409

34 Tenson, T. and Ehrenberg, M. (2002) Cell 108, 591-594

35 Jakoby, M., Weisshaar, B., Droge-Laser, W., Vicente-Carbajosa, J., Tiedemann, J., Kroj, T. and Parcy, F. (2002) Trends Plant Sci. 7, 106-111

36 Wiese, A., Elzinga, N., Wobbes, B. and Smeekens, S. (2004) Plant Cell 16, 1717-1729

37 Farrar, J., Pollock, C. and Gallagher, J. (2000) Plant Sci. 154, 1-11

38 Hill, L.M., Morley-Smith, E.R. and Rawsthorne, S. (2003) Plant Physiol. 131, 228-236

39 Borisjuk, L., Rolletschek, H., Wobus, U. and Weber, H. (2003) J. Exp. Bot. 54, 503-512

40 Farrar, S.C. and Farrar, J. (1987) Plant Physiol. Biochem. 25, 541-548

41 Rook, F., Weisbeek, P. and Smeekens, S. (1998) Plant Mol. Biol. 37 $171-178$

42 Lee, S.J., Lee, M.Y., Yi, S.Y., Oh, S.K., Choi, S.H., Her, N.H., Choi, D., Min, B.W., Yang, S.G. and Harn, C.H. (2002) Mol. Plant Microbe Interact. 15, 540-548

43 Satoh, R., Fujita, Y., Nakashima, K., Shinozaki, K. and Yamaguchi-Shinozaki, K. (2004) Plant Cell Physiol. 45, 309-317

44 Kusano, T., Berberich, T., Harada, M., Suzuki, N. and Sugawara, K. (1995) Mol. Gen. Genet. 248, 507-517

45 Yang, S.H., Berberich, T., Sano, H. and Kusano, T. (2001) Plant Physiol. 127, 23-32

46 Aguan, K., Sugawara, K., Suzuki, N. and Kusano, T. (1993) Mol. Gen. Genet. 240, 1-8

47 Strathmann, A., Kuhlmann, M., Heinekamp, T. and Droge-Laser, W. (2001) Plant J. 28, 397-408

Received 23 August 2004 